\title{
Ecological Sucking Monitoring of Newborns
}

\author{
Fabrizio Taffoni, Member, IEEE, Eleonora Tamilia, Member, IEEE, Maria Rosaria Palminteri, \\ Emiliano Schena, Member, IEEE, Domenico Formica, Member, IEEE, Jonathan Delafield-Butt, Flavio \\ Keller, Sergio Silvestri Member, IEEE, Eugenio Guglielmelli, Senior Member, IEEE.
}

\begin{abstract}
Feeding by sucking is one of the first activities of daily life performed by infants. Sucking plays a fundamental role in neurological development and may be considered a good early predictor of neuromotor development. In this work a new method for ecological assessment of infants' nutritive sucking behaviour is presented and experimentally validated.

Preliminary data on healthy newborn subjects were first acquired to define the main technical specifications of a novel instrumented device. This device was designed to be easily integrated in a commercially available feeding bottle, allowing clinical methods development for screening large numbers of subjects.

The new approach proposed allows (i) accurate measurement of intra-oral pressure for neuromotor control analysis and (ii) estimation of milk volume delivered to the mouth to within less than $2 \%$ variation between estimated and reference volumes.
\end{abstract}

Index Terms - Instrumented objects, Suckling Monitoring, Ecological Assessment, Nutritive sucking, Motor control

\section{INTRODUCTION}

$\mathrm{N}$ EUROPHYSIOLOGICAL development in infants may be indirectly assessed by the study of movement. Precthl demonstrated spontaneous motility of infants could be regarded as the expression of spontaneous neural activity, presenting an excellent marker of neural dysfunctions [1]. Early motor acts and their development provide the infant with new experiences and opportunities for exploration of the world, and world exploration in turn creates context for the development of the brain [2]. This process is critical, since the progressive acquisition of motor skills provides infants with an increasingly growing set of opportunities for acquiring, practicing and refining abilities, that is an essential prerequisite for growth in other domains, especially those of

Manuscript received March 05, 2013. This work was partly founded by the Italian Ministry of Education, University and Research under the FIRB "Futuro in Ricerca" research program (TOUMproject, no. B81J10000160008).

Fabrizio Taffoni, Eleonora Tamilia, Maria Rosaria Palminteri, Domenico Formica, and Eugenio Guglielmelli are with the Laboratory of Biomedical Robotics and Biomicrosystems, Università Campus Bio-Medico di Roma, Via Alvaro del Portillo, 28, 00128, Rome, Italy (e-mail: \{f.taffoni; e.tamilia; m.palminteri; d.formica; e.guglielmelli\}@unicampus.it).

Emiliano Schena and Sergio Silvestri are with the Center for Integrated Research, Unit of Measurements and Biomedical Instrumentation, Università Campus Bio-Medico di Roma, Via Alvaro del Portillo, 28, 00128, Rome, Italy (e-mail: \{e.schena;s.silvestri\}@unicampus.it).

Jonathan Delafiel-Butt, Perception Movement Action Consortium, University of Edinburgh \& Early Years, University of Strathclyde, Glasgow G4 0LT, Scotland, U.K. (e-mail: jonathan.delafield-butt@strath.ac.uk).

Flavio Keller is with Laboratory of Developmental Neuroscience and Neural Plasticity, Università Campus Bio-Medico di Roma, Via Alvaro del Portillo, 28, 00128, Rome, Italy (e-mail: f.keller@unicampus.it). language and social interaction [3]. Therefore, the investigation of early motor skills in newborns provides a window not only on the development of motor control, but more generally on the neural dynamics that underpin neuropsychological health for development and learning [4]. Instruments and methods typically used to investigate learning and also re-learning capabilities in human adult subjects [5][7] are not suitable for newborn infants because of their obtrusiveness and complex equipment. Recently developed instrumented toys and other objects, possibly embedding purposively developed microsensors [8][9], offer a great possibility for ecological non-obtrusive investigation of brain development in infancy and childhood [10]-[14]. Despite current researches on novel design methodologies for advanced wearable systems [15], such technologies are not suitable for newborn infants with their comparatively poor motor repertoires and relative disinterest in manipulating objects. Sucking, on the other hand, is one of the first and most frequent action performed by infants [16][17]. It begins in uterus from gestational age 15 to 18 weeks [18] and usually becomes stable and well patterned by 34 weeks in preparation for birth [19].

Nutritive sucking (NS) postpartum allows an infant to obtain food. It consists of a series of bursts and pauses; each burst contains several suck cycles that occur at approximately $1 \mathrm{~Hz}$ [20]. A nutritive suck is characterized by the alternation of suction and expression [21]: suction defines the negative intraoral pressure generated by the infant in order to draw milk into the mouth and expression corresponds to the compression and/or stripping of the nipple between the tongue and the hard palate as milk is ejected into the mouth [22][23].

The act of sucking is a complex task for the neonate, because it requires not only an efficient sucking ability, but also a proper coordination between the rhythmic processes of sucking, swallowing and respiration [24]-[26]. This coordination requires intact brainstem pathways and transmission of impulses through the cranial nerves to healthy musculature in the mouth, tongue, and pharynx [27]. Lack of coordination explains changes in the sucking rate and the appearance of abnormal clinical signs such as low consumption of food, choking, regurgitation, vomiting or respiratory disorders. For this reason, the assessment of the sucking pattern represents one of the most accessible and earliest possible cues of infant neurophysiological status, as shown for the first time by Wolff [20], followed by several others [26]-[29]. Precise analyses of sucking can provide not 
only valuable insights into the integrity of an infant's central nervous system, but can also serve as an early prognostic indicator of future neurodevelopmental disorder. Mizuno et al. [27] found that patterns and values of expression and sucking pressures during neonatal feeding can predict neurodevelopmental outcomes evaluated by the Bayley Scales of Infant Development (BSID) at 18 months. Medoff-Cooper [30] as well reported a significant relationship between sucking organization (characterized only by sucking pressure values) and BSID values, evaluated at the earlier age of 12 months. Craig et al. [31] reported evidence that the degree of control of the increasing and decreasing suction pressures generated near corrected term in a prematurely-born 'at risk' cohort predicted motor development measured at 6 months using postural, fine, and gross motor assessment.

These studies stimulate further development of protocols for neonatal sucking assessment as an indicator of infants' neurophysiological health or dysfunction, and may help to address an unmet need for a simple, cost-effective screening strategy for early identification of infants at risk for cognitive and psychomotor delays [32].

Hence, driven by this need, the aim of this study has been to develop a new methodology for an objective measurement of sucking parameters and to integrate such a methodology in a portable, non-intrusive, low-cost device.

\section{Functional AND TeChNicAl Specifications}

Analysis of intra-oral pressure changes during newborn NS may represent one of the earliest easily accessible assessments of infant neurophysiological status [30]. Further, simultaneous assessment of the volume of ingested milk may allow quantification of the NS efficiency for improved assessment [33]. Yet, the methodological solutions previously adopted, i.e. in the NS studies cited above, were based on complex, non-portable technologies instrumented with ultrasound systems, physiological pressure monitoring, or with additional equipment for continuous measurement by weight of the milk reservoir. These systems previously employed were complex, expensive, and awkward to deploy. They remain restricted to small-scale research studies and do not allow for extended application to large-scale studies or deployment to everyday domestic or clinical settings. Such deployment is desirable for methodological and analytical improvements that may ultimately contribute significant new data to clinical assessment of infant neurophysiological health. Two principal modalities of data on infant sucking are apparent: (i) continuous monitoring of intra-oral pressure and (ii) continuous monitoring of expressed milk volume. For these reasons the proposed device should monitor intra-oral pressure and volume of taken milk in an ecological, unobtrusive way, even at home during regular bottle feeding. For ecological we mean a methodology and/or an instrumentation that don't modify the environment and/or the observed task. In the feeding task, for example, the inclination of the bottle with respect to gravity is particularly important because low inclination may not completely fill the nipple with milk, while, in case of high inclination $\left(>60^{\circ}\right)$, the infant may experience high hydrostatic pressure, especially at the beginning of feeding. Emphasis on ecological nature of data collection is common in behavioral studies and becomes particularly important for infants who may already be distressed and can not cooperate with experimenters or clinicians with additional, complex and time-consuming settings and procedures.

For example, another possibility for measuring the milk volume ingested is to use some simple non-technological approaches, such as weighing the bottle before and after the feed or checking the volume of ingested milk by visual inspection of the scale on the side of the bottle. The main drawback of these solutions is that they allow a global estimation of the ingested volume, but they do not continuously assess milk volume intake; therefore, they do not allow an energetic analysis of sucking. Simultaneous recording of both intraoral pressure and milk volume intake overcomes this limitation [34]. Moreover, the assessment of the rate of milk flow during bottle feeding it is known to play a crucial role for clinical evaluation: it is one of the most important determinants of feeding-related ventilator changes in some term and preterm infants, where the efficient feeding and breathing assistance is crucial for physiological and development health [35].

In order to define the specifications of the device that should simultaneously and continuously measure intra-oral pressure and milk volume intake preliminary data analysis was undertaken to verify intra-oral pressure range and bandwidth. Eight term-birth, healthy infants were recorded while feeding shortly after birth (mean age 2.6 days, std. dev. 1.5 days) and four were recorded approximately 8 weeks later (mean age 55 days, std. dev. 15 days). Intra-oral pressure was measured by inserting an umbilical catheter $40 \mathrm{~cm}$ in length with an internal diameter of $1.0 \mathrm{~mm}$ and external diameter of $1.7 \mathrm{~mm}$ (Ref. 1270.05, Vygon, France) through the teat (Standard Teat, Cow \& Gate, UK) of a feeding bottle so that it protruded approximately $2 \mathrm{~mm}$ from its tip into the middle of the infant's oral cavity. The catheter was primed with distilled water and connected to a pressure transducer (TranStar, Medex, UK/France/Italy/Germany) and medical physiology monitor (Datex-Ohmeda Light, Finland). Data were captured to a laptop PC using proprietary software (Collect4, General Electric Healthcare, Finland).

Fig. 1 reports a typical 10 seconds pressure burst: experimental data confirm that intra-oral pressure is in the range of $[-140+15] \mathrm{mmHg}$ reported in literature [36]. The bandwidth of the pressure signal has been estimated calculating its Power Spectral Density (PSD) by means of the Welch overlapped segmented average. As shown by Fig. 2 the bandwidth may be considered well below $20 \mathrm{~Hz}$, which has been taken as the required bandwidth of our device. The volume of milk ingested by an infant during each suck or burst may depend on several factors, e.g., the infant's age, weight, postconceptional age (PCA) at birth.

Table I reports the values of several sucking parameters obtained by several authors in their studies [24],[33][36]-[38]. The values reported by Taki et al. in their recent study [36] have been considered in the present work because they report both the volume of a single suck and the volume of an entire burst, unlike Fadavi's [37] and Lang's [38] works, and 
because they also report on longitudinal sucking performance, allowing for consideration of differences from 1 to 6 months of age.

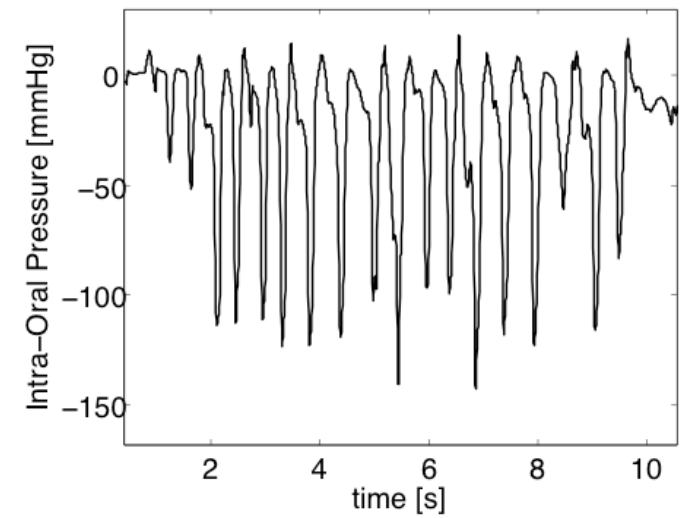

Fig. 1 Intra-oral pressure of a 1 week newborn recorded during a feeding task.

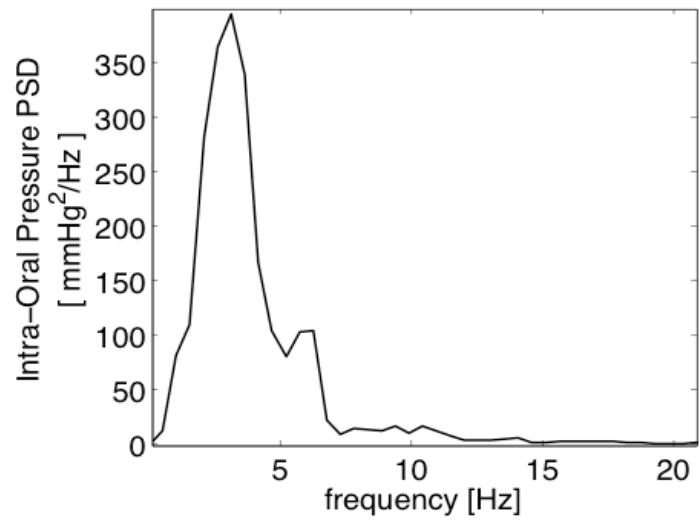

Fig. 2 Power Spectral Density of intra-oral pressure during sucking. In the example reported above the PSD is below $20 \mathrm{~Hz}$. The peak below $5 \mathrm{~Hz}$ is due to Nutritive Sucking (NS) whereas the peak at about $6 \mathrm{~Hz}$ is due to breathing.

TABLE I

VALUES OF PARAMETERS RELATED TO SUCKING: REVIEW OF THE MAIN

\begin{tabular}{llllll}
\hline \multicolumn{1}{c}{$\begin{array}{c}\text { First } \\
\text { Author }\end{array}$} & $\begin{array}{c}\text { PCA at } \\
\text { birth } \\
\text { week } \\
(\mathrm{M} \pm \mathrm{SD})\end{array}$ & $\begin{array}{c}\text { PNA } \\
{[\mathrm{d}]}\end{array}$ & $\begin{array}{c}\text { Sucks/min } \\
(\mathrm{M} \pm \mathrm{SD})\end{array}$ & $\begin{array}{c}\text { Vol/suck } \\
{[\mathrm{mL}]} \\
(\mathrm{M} \pm \mathrm{SD})\end{array}$ & $\begin{array}{c}\text { Sucks/burst } \\
(\mathrm{M} \pm \mathrm{SD})\end{array}$ \\
\hline Fadavi & Term & 2 & $73 \pm 14$ & $0.056 \pm 0.016$ & --- \\
1997 & & & $65 \pm 9$ & $0.113 \pm 0.039$ & \\
{$[37]$} & & & $72 \pm 8$ & $0.067 \pm 0.031$ & \\
& & & $71 \pm 9$ & $0.077 \pm 0.024 *$ & \\
\hline Qureshi & $39.2 \pm 1.1$ & $1-4$ & 55 & $0.235 \pm 0.102$ & $10 \pm 9$ \\
2002 & $43.2 \pm 1.1$ & 30 & 70 & $0.343 \pm 0.123$ & $21 \pm 22$ \\
{$[33]$} & & & & & \\
\hline Taki & $39.9 \pm$ & 30 & --- & $0.28 \pm 0.16$ & $38 \pm 12.2$ \\
2010 & 1.1 & 90 & & $0.30 \pm 0.07$ & $43 \pm 7$ \\
{$[36]$} & & 120 & & $0.40 \pm 0.24$ & $82 \pm 22$ \\
\hline Macias & Full- & --- & $55(18-$ & $0.8-1.2$ & $20-30$ \\
2011 & term & & $100)$ & & $10-20$ \\
{$[24]$} & & & & & $3-10$ \\
\hline Lang & Full- & $38-$ & $1.143 / \mathrm{s}$ & --- & 122 \\
2011 & term & 47 & 1.132 & & 107 \\
{$[38]$} & & PMA & 1.17 & & 88 \\
& & & 1.195 & & 78 \\
& & & & &
\end{tabular}

*Different values obtained with different nipple
According to [36], a bottle-fed infant ingests $0.28 \pm 0.13 \mathrm{~mL}$ per suck at the postnatal age of 1 month. This value slightly increases with the age, reaching $0.30 \pm 0.24 \mathrm{~mL}$ at 3 months and $0.40 \pm 0.20 \mathrm{~mL}$ at 6 months. These values, together with the number of sucks per burst, allow estimation of the minimum volume of milk an infant can ingest in a suck or a burst. During the first 6 months of life the average value of $0.2 \mathrm{~mL}$ has been considered a minimum volume per suck, whereas $8 \mathrm{~mL}$ is considered the minimum volume per burst.

\section{Design And FABRicAtion of Suction DEVICE}

\section{A. General Architecture}

The two main functional requirements for the sucking device are its portability and ease of use. For this reason, a smart module was designed and developed to integrate into a commercial infant feeding bottle. Fig. 3 reports the general architecture of the module. A key idea was to physically decouple the sensing electrical part from the milk reservoir. To this end the electronics were separated from the milk reservoir by a semipermeable membrane (this allows air flow, but blocks the liquid) placed between the reservoir and the electronics: direct contact between the milk and the electronics was avoided, solving both important problems of contamination and electrical safety.

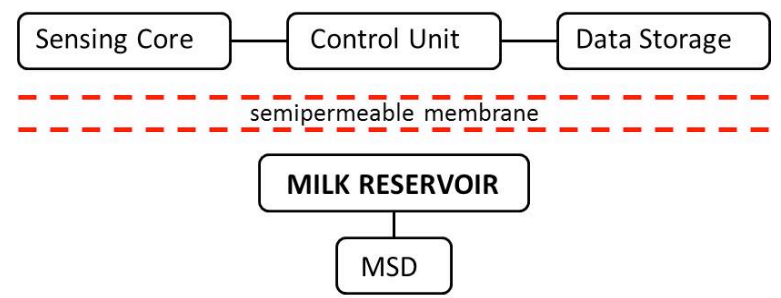

Fig. 3 The general architecture of the device. Electronic components are separated from the milk reservoir thanks to a membrane. A Mechanical Safety Device (MSD) is added to the milk reservoir to avoid the creation of excessive negative pressures inside the bottle higher beyond a threshold level.

The sensing core had to integrate sensors allowing measurement of intra-oral pressure and volume of ingested milk. The components of the sensing core will be discussed in detail in Section III.B below. Data coming from sensing core needed to be acquired by the control unit and stored on a suitable support. To allow portability, robustness and guarantee long duration monitoring, a micro SD support was chosen. For this reason the control unit was required have the RAM necessary for writing/reading operations on a micro SD: for FAT16 micro-SD, 512 MB RAM memory was deemed necessary and a PIC18F46J50 chosen to be used.

Finally, a mechanical safety device was provided on the bottom of the bottle in order to avoid conditions that may hinder the infant's sucking. This is illustrated in Section III.C below.

\section{B. Sensing Core}

The intraoral sucking pressure is measured with a modified disposable teat connected via a non-compliant catheter (1.2 $\mathrm{mm}$ of internal diameter) to a low cost integrated silicon 
pressure sensor (MPX7025, Freescale Semiconductor). This sensor has a range of $\pm 25 \mathrm{kPa}$ (about $\pm 190 \mathrm{mmHg}$ ), well above the physiological range of intraoral pressures (Fig. 1).

The catheter was inserted into the teat through an incision at its base. The tube was then threaded into the inside of the teat and out through one of the three manufactured feeding holes at the top. To be able to measure the intraoral sucking pressure, the tube was positioned so that it protruded about $2 \mathrm{~mm}$ through the end of the teat as reported in [37].

The estimation of the volume of milk delivered to the infant during each sucking act is particularly important because it allows quantification of the effectiveness of each NS.

It is very common in pediatric practice to weigh the bottle at the beginning and at the end of the feeding to obtain a raw estimation of the overall amount of milk delivered to the child. In the above cited researches, authors provided complex measurement systems to refine this technique. The proposed systems are quite complex and expensive. In this work we propose a low cost method to estimate the volume of milk delivered to the newborn based on a measurement of pressure. During a single suck, an infant closes her/his lips around the bottle teat and, as the jaw and tongue drop down, produces a negative pressure inside the oral cavity that causes the milk flow towards the mouth. In this phase, as long as a good seal around the teat is maintained, no air can flow inside the bottle. The flow of milk toward the mouth causes the generation of a gradual negative pressure inside the bottle which persists until the newborn releases her/his lips allowing air to flow inside the bottle. Therefore, by measuring the pressure of a gas (the air inside the bottle), an estimation of the milk volume delivered to the infant during each suck may be obtained, as reported in other medical fields [39].

In general, the relationship between volume and pressure of a gas may be expressed as a function of number of moles (n), gas volume $(\mathrm{V})$ and temperature $(\mathrm{T})$ :

$$
P=P(n, V, T)
$$

Taking into account all the different variables, the pressure drop (dP) within the bottle can be expressed as:

$$
d P=\frac{\partial P}{\partial n} d n+\frac{\partial P}{\partial V} d V+\frac{\partial P}{\partial T} d T
$$

During each suck, the number of moles of the gas inside the bottle may be considered constant because there is not air exchange between the bottle and the environment $(\mathrm{dn}=0)$. Moreover, variation of air temperature may be hypothesized as negligible during a suck. Taking into account these assumptions, and considering the air inside the bottle as an ideal gas $(P V=n R T)$, equation (2) may be rewritten to estimate the variation of air volume $(\mathrm{dV})$ inside the bottle as:

$$
d V \cong-\frac{V^{2}}{n R T} d P
$$

$\mathrm{V}$ in this equation represents the volume of air inside the bottle before the suck. This volume may be updated using a recursive algorithm that increments its value by the estimate dV. Referring to Fig. 4, it will be:

$$
\begin{aligned}
& V_{1}=V_{0}+d V_{0} \\
& V_{2}=V_{1}+d V_{1} \\
& \cdots \\
& V_{i+1}=V_{i}+d V_{i}
\end{aligned}
$$

where $\mathrm{V}_{0}$ is the initial volume of air inside the bottle. The change in volume of air for each suck will be equal (disregarding the sign) to the volume of milk delivered to the child.

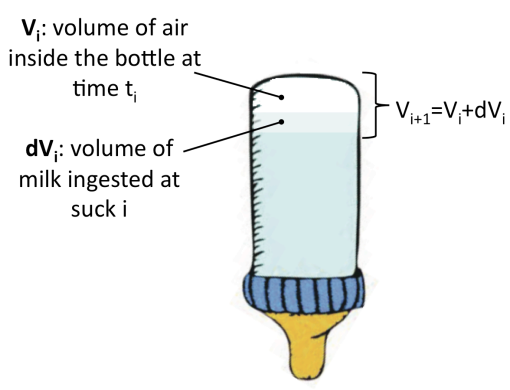

Fig. 4 Feeding bottle: at time $t_{i}$ there is an inner air volume equal to $V_{i}$; after one suck, a volume variation equal to $\mathrm{dV}_{\mathrm{i}}$ will be obtained.

The term nRT in absence of air flow may be considered constant and equal to the product between $\mathrm{P}$ and $\mathrm{V}$ (according to the Boyle-Mariotte's law), for this reason (3) may be expressed as:

$$
d V=\frac{V}{P} d P
$$

$V_{0}$ may be estimated producing a perturbation of known volume $(\Delta V)$ and measuring the air pressure before $\left(P_{1}\right)$ and after $\left(P_{2}\right)$ the volume variation. It will be:

$$
\begin{gathered}
P_{1} V_{0}=A \\
P_{2}\left(V_{0}+\Delta V\right)=A
\end{gathered}
$$

From the previous ones, the unknown $v_{0}$ will be:

$$
V_{0}=\frac{P_{2} \Delta V}{P_{1}-P_{2}}
$$

in this way a semiautomatic initialization of the device will be possible enabling its use ecologically in non-structured environments. In order to estimate the volume of milk delivered to the infant during the sucking acts, using the method described above, the sensing core has been equipped with a second pressure sensor, MPX7025.

\section{Integration}

The sensing core has been integrated on a commercially available infant feeding bottle. For solving the contamination and electrical safety problems, described in section III.A, a commercial bottle was used (First Bottle, MAM Babyartikel GesmbH, Vienna, Austria) with a vented base with eight air holes and an embedded silicone membrane that blocks liquid but not air, allowing an actual separation of the electronic smart part from the milk reservoir. The vented base was experimentally modified to prevent air entering into the bottle 
as milk flowed out, to satisfy the method described in section III.B. Hence, all the holes except one (the one that will be needed for the intra-bottle pressure measurement) were sealed with a non-toxic smooth consistency mouldable silicone rubber (RTV-530, Prochima S.r.l.). Due to this modification, if the newborn does not release his/her lips around the teat, a decreasing pressure inside the bottle will generate. If such pressure reaches the equilibrium with the opposing suction pressure exerted by the infant, milk flow may be hampered [40].
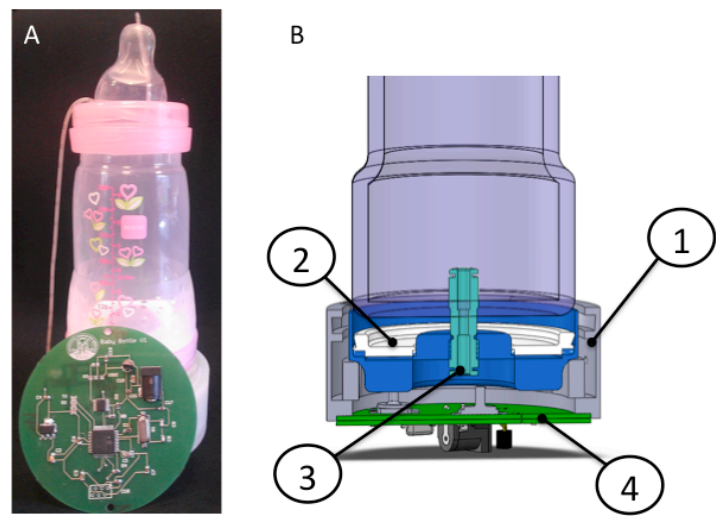

Fig. 5 Instrumented feeding bottle: A) prototype; B) 3D-CAD of the prototype. In $\mathrm{B}$ it is possible to observe: 1 external electronic support, 2 silicon membrane; 3 MSD; 4 electronics

Since such conditions would alter the infant's sucking behavior, an additional mechanical safety device has been included at the bottle base, with the aim of preserving the newborn from excessive fatigue rather than estimating the volume of milk taken in under such altered conditions. The device is a polypropylene check valve (Check Valve PP6952B2BF, Coast Pneumatics Inc.) with a $60 \mathrm{mmHg}$ cracking pressure; it allows the air to flow inside the bottle when the negative pressure inside it exceeds the value of $-60 \mathrm{mmHg}$. A series of tests have been carried out on the valve, showing that $1 \mathrm{~s}$ after its opening the mean intra-bottle pressure increase is $10.7 \pm 0.4 \mathrm{mmHg}$, preventing in this way the negative pressure inside the bottle to rise above values that can cause excessive fatigue to the infant, in case she/he does not release the teat.

To allow for bottle sterilization in between uses, an external support for the electronics has been designed. This support consists of two parts connected through a bayonet closure: one part is attached to the bottle base, whereas the other one, including the electronics, can be easily removed (see Fig. 5) allowing for easy washing and sterilization of the bottle.

\section{EXPERIMENTAL VALIDATION}

Experimental validation of the method for volume estimation proposed in section III.B has been carried out as described below. The experimental setup used is shown in Fig. 6. A graduated reservoir was filled with water at room temperature. The reservoir was connected to the atmosphere by means of a manual on/off valve. The pressure sensor MPXV7025 is used to measure the air pressure. The base of the reservoir is connected by means of three-way valve to a syringe $(\operatorname{Vmax}=1 \mathrm{~mL}$, resolution $0.01 \mathrm{~mL}$ ). The manual on/off valve simulates the child's lips: when the lips are closed around the teat the valve is closed, when the lips are released, allowing air flow inside the bottle, the valve is opened. The syringe is used to simulate suck and burst allowing intake from $0.1 \mathrm{~mL}$ to $1 \mathrm{~mL}$ of liquid.
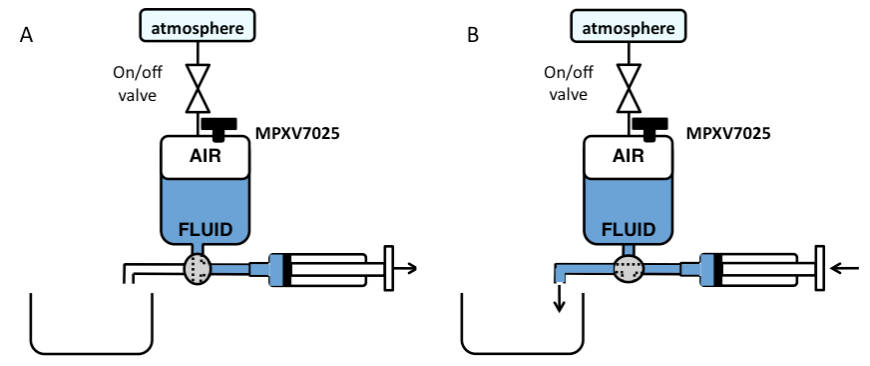

Fig. 6 Experimental setup used for empirical validation of volume estimation method presented in section III.B

According to (5) and for a fixed $\Delta V$, the measured pressure variation is inversely proportional to the inner air volume. For this reason, the maximal inner air volume compatible with pressure sensor resolution (about $1 \mathrm{mmHg}$ ) needed to be estimated. A fixed volume of water equal to 1 suck $(0.2 \mathrm{~mL})$ was subtracted for ten times to the reservoir and the corresponding pressure drop measured. After each subtraction, the water was reinserted into the reservoir and the on/off valve opened to allow atmospheric pressure in the chamber. Trials have been repeated at different initial air volumes: $25,35,45$, $55,65 \mathrm{~mL}$, to simulate the reduction of liquid inside the bottle due to nutrition. Results of these trials are reported in Fig. 7: as reported in (5), the pressure drop was inversely proportional to the inner air volume and may be fitted with a power law $\left(f(x)=a x^{\wedge} b, a=167.3, b=-1.034, R 2=0.99\right)$. According to this fit, the maximal inner volume compatible with pressure sensor resolution was equal to $140 \mathrm{~mL}$, corresponding to a volume of ingested milk equal to $115 \mathrm{~mL}$ (almost two times higher than volume of milk ingested by healthy newborns).

In order to assess the performances of the proposed method, a set of measurements at the same inner air volumes reported above was carried out, for a total variation of liquid equal to $40 \mathrm{~mL}$. Ten preliminary measurements with $25 \mathrm{~mL}$ of air were performed to estimate the initial V0 according to (7). The estimated volume was $25.59 \pm 0.07 \mathrm{~mL}$ (percent error $=$ $2.4 \%$ ). For each inner air volume $0.2 \mathrm{~mL}$ of liquid (equal to 1 suck) was subtracted by the reservoir (see Fig. 6.A), then the reservoir was closed using the three way valve and the syringe emptied in an external tub (see Fig. 6.B). After 15 sucks the on/off valve was opened simulating the releasing of the lips around the teat and the consequent air flow allowed inside the bottle. For each suck, the volume of subtracted liquid was estimated according to (3) and compared with the actual subtracted volume. Trials were repeated at different inner air volumes to verify if the reduction of sensitivity may affect the estimation of volume in the considered range (see Table II).

In Fig. 8 volume estimation results (blue points) are compared with the actual volume subtracted (red dotted line). Despite decrease of sensitivity with increasing inner air volume, the 
error remains small and does not exceed the $2 \%$ of the actual volume subtracted.

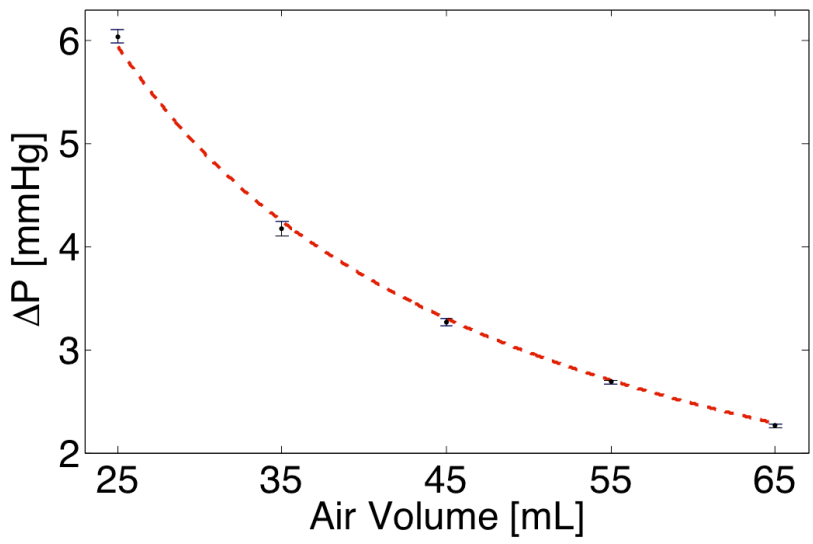

Fig. 7 Pressure variation for different air volumes inside the bottle. The subtracted liquid volume is equal to $0.2 \mathrm{~mL}$. The dotted red line represents the power regression model.

TABLE II

$\triangle$ V ESTIMATION AT DIFFERENT INNER AIR VOLUMES

Inner Air Volume $[\mathrm{mL}] \quad$ Estimated $\Delta \mathrm{V}[\mathrm{mL}]$

$\begin{array}{ll}25 & 0.204 \pm 0.003 \\ 35 & 0.197 \pm 0.002 \\ 45 & 0.198 \pm 0.002 \\ 55 & 0.200 \pm 0.002 \\ 65 & 0.204 \pm 0.004\end{array}$

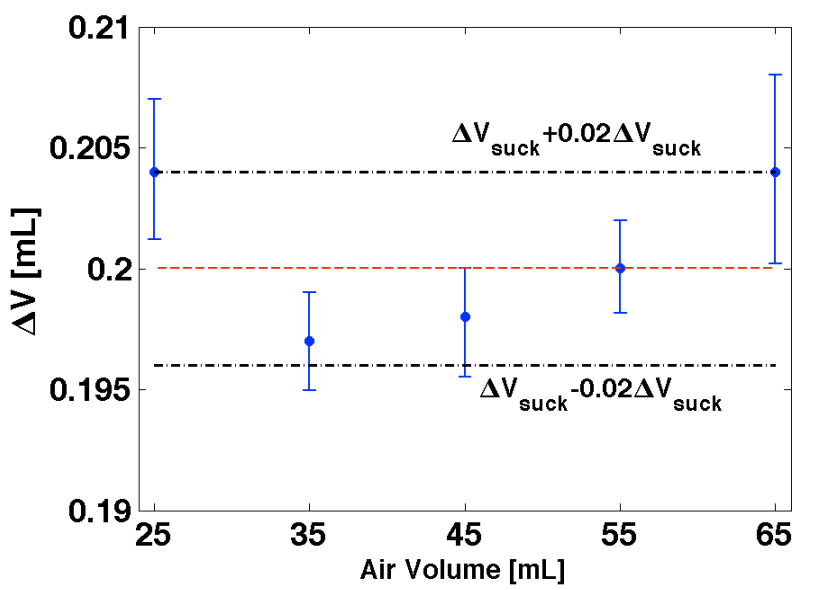

Fig. 8 Volume variation estimation for different air volume inside the bottle. The dotted red line represents the actual liquid volume variation, equal to 0.2 $\mathrm{mL}$. The dash-dotted black lines represent the actual value $\pm 2 \%$

\section{CONCLUSION AND FUTURE WORK}

The act of sucking is a complex task for newborns, requiring necessary strength of all oropharyngeal structures involved as well as adequate maturation of the relevant motor circuits within the central nervous system. For this reason it represents a good candidate for indirect assessment of neurophysiological development of newborns.
In this work, a new methodological advance for newborn sucking monitoring has been presented and experimentally validated. A low-cost electronic has been designed and integrated into a commercially available bottle. The prototype instrument allows continuous measurement of intraoral pressure and continuous estimation of volume of milk delivered to the newborn, to within a $2 \%$ margin of error, during feeding. Volume estimation is based on measurement of the air pressure inside the feeding bottle. Moreover, volume estimations are independent from the tilt of the bottle with respect to gravity, so that it is not needed any orientation monitoring, or keeping the bottle at a specific angle to obtain good volume estimations, as done by [41]. Since the use of our new device does not modify how bottle feeding is commonly done (e.g. by altering the tilting angle) and since it does not need complex calibration procedures, this new approach may be used by untrained personnel in everyday domestic and clinical settings. Thus, it may represent an ecologically valid, cost-effective, and accessible approach enabling large scale, continuous monitoring of infant NS. This kind of studies will, in turn, enable the assessment of the development of NS, and its deviation in cases of pathologies.

After approval by an ethical committee, a pilot study on a small number of newborns will be carried out to verify the performance of the proposed technology in the field.

\section{REFERENCES}

[1] H.F.R. Prechtl, "State of the art of a new functional assessment of the young nervous system. An Early predictor of cerebral palsy.", Early Hum. Dev., vol. 50, Nov. 1997, pp. 1-11.

[2] E. Thelen, "Motor development as foundation and future of developmental psychology.", Int. J. of Behav. Dev., vol. 24(4), Dec. 2000, pp385-397.

[3] J.M. Iverson, " Developing language in a developing body: The relationship between motor development and language development", Journal of Child Language, Jan. 2010, vol. 37, pp. 229-261.

[4] C. von Hofsten, "Action in development.", Dev. Sci., vol.10(1), Jan. 2007, pp 54-60.

[5] D Formica, SK Charles, L Zollo, E Guglielmelli, Hogan, N., Krebs, H.I, "The passive stiffness of the wrist and forearm", Journal of Neurophysiology, 2012, Volume 108(4), pp. 1158-1166.

[6] D Formica, L Zollo, E Guglielmelli Torque-dependent compliance control in the joint space for robot-mediated motor therapy", Journal of dynamic systems, measurement, and control, Volume 128, 2006, Pages 152-158.

[7] Pellegrino, G., Pellegrino, G., Tombini, M., Assenza, G., Bravi, M., Sterzi, S., Giacobbe, V., Zollo, L., Guglielmelli, E., Cavallo, G., Vernieri, F., Tecchio, F., "Inter-hemispheric coupling changes associate with motor improvements after robotic stroke rehabilitation", Restorative Neurology and Neuroscience, Volume 30, Issue 6, 2012, Pages 497-510.

[8] Accoto D., Sahai R., Damiani F., Campolo D., Guglielmelli E., Dario P. "A slip sensor for biorobotic applications using a hot wire anemometry approach", Sensors and Actuators, A: Physical, 187 , 2012, pp. 201-208.

[9] M. T. Francomano, D. Accoto, E. Morganti, L. Lorenzelli, E. Guglielmelli, "A microfabricated flexible slip sensor", Proceedings of the IEEE RAS and EMBS International Conference on Biomedical Robotics and Biomechatronics, 2012, pp. 1919-1924.

[10] F.Taffoni, C. von Hofsten, "Tact Glossary: Toy", Clin. Ter., vol. 161(6), 2010, pp. 573-574.

[11] D. Campolo, F. Taffoni, D. Formica, G. Schiavone, F. Keller, E. Guglielmelli, "Inertial-Magnetic Sensors for Assessing Spatial Cognition in Infants", IEEE Trans. Biomed. Eng., vol.58(5), 2011, pp. 1499-503.

[12] D. Campolo, F. Taffoni, D.Formica, J.Iverson, L. Sparaci, F.Keller, E.Guglielmelli, "Embedding Inertial-Magnetic Sensors in Everyday 
Objects: Assessing Spatial Cognition in Children", J. Integr. Neurosci., vol. 11(1), Mar. 2012, pp.103-116.

[13] F. Taffoni, D. Formica, A. Zompanti, M. Mirolli, G. Balsassarre, F. Keller,.E. Guglielmelli, "A mechatronic platform for behavioral studies on infants," Biomedical Robotics and Biomechatronics (BioRob), 2012 4th IEEE RAS \& EMBS International Conference on, pp.1874,1878, 2427 June 2012.

[14] F. Taffoni, V. Focaroli, D. Formica, J. M. Iverson, F. Keller, E. Gugliemelli "Sensor-based technology in the study of motor skills in infants at risk for ASD," Biomedical Robotics and Biomechatronics (BioRob), 2012 4th IEEE RAS \& EMBS International Conference on , vol., no., pp.1879,1883, 24-27 June 2012

[15] F. Sergi, D. Accoto, N. L. Tagliamonte, G. Carpino, E. Guglielmelli, "A systematic graph-based method for the kinematic synthesis of nonanthropomorphic wearable robots for the lower limbs", Frontiers of Mechanical Engineering, Vol. 6 (1), 2011, pp. 61-70.

[16] H. Forssberg, "Neural control of human motor development.", Curr Opin Neurobiol vol. 9, Dec. 1999, pp. 676-682.

[17] A. Kurjak, Stanojevic, M., Andonotopo, W., Salihagic-Kadic, A., Carrera, J. M., Azumendi, G. "Behavioral pattern continuity from prenatal to postnatal life-A study by four-dimensional (4D) ultrasonography." Journal of Perinatal Medicine, 32, 2004, 246-253.

[18] J. L. Miller, B. C. Sonies, C. Macedonia, "Emergence of oropharyngeal, laryngeal and swallowing activity in the developing fetal upper aerodigestive tract: An ultrasound evaluation.”, Early Human Development, 71, 2003, pp. 61-87.

[19] M. Hack, M. M. Estabrook, S. S. Robertson, "Development of sucking rhythm in preterm infants." Early Human Development, 11, 1985, pp. $133-140$.

[20] P. H. Wolff, "The serial organization of sucking in the young infant." Pediatrics, 42, 1968, pp. 943-956.

[21] A. J. Sameroff , "The components of sucking in the human newborn." $J$ Exp Child Psychol, 6, 1968, pp. 607-23.

[22] R. A. Waterland, R. I. Berkowitz, A. J. Stunkard, V. A. Stallings, "Calibrated-orifice nipples for measurement of infant nutritive sucking." J Pediatr, 132, 1998, pp. 523-6.

[23] A. J. Nowak, W. L. Smith, A. Erenberg, "Imaging evaluation of artificial nipples during bottle feeding." Arch Pediatr Adolesc Med, 148, 1994, pp. $40-2$.

[24] M. E. R. Macías, G. J. S. M. Meneses, "Physiology of nutritive sucking in newborns and infants", 68(4), 2011, pp. 296-303.

[25] C. Lau, "Oral Feeding in the Preterm Infant." NeoReviews, 7(1), 2006, pp. e19-e27.

[26] K. Mizuno, A. Ueda, "The maturation and coordination of sucking, swallowing, and respiration in preterm infants." The Journal of pediatrics, 142(1), 2003, pp. 36-40.

[27] K. Mizuno, A. Ueda, "Neonatal feeding performance as a predictor of neurodevelopmental outcome at 18 months." Developmental medicine and child neurology, 47(5), 2005, pp. 299-304.

[28] I. H. Gewolb, J. Bosma, E. Reynolds, F. Vice, "Integration of suck swallow rhythms during feeding in preterm infants with and without bronchopulmonary dysplasia." Developemental and Behavioral Pediatrics, 45, 2003, pp. 344-348.

[29] B. Medoff-Cooper, W. Bilker, J. Kaplan, "Suckling behavior as a function of gestational age: A cross-sectional study. Infant Behavior and Development, 24, 2001, pp. 83-94.

[30] B. Medoff-Cooper, J. Shults, J. Kaplan, "Sucking Behavior of Pre-term Neonates As a Predictor of Developmental Outcomes", 2008, pp. 1-22.

[31] C.M. Craig, M.A. Grealy \& D.N. Lee, "Detecting motor abnormalities in preterm infants", Exp. Brain Res., Vol 131(3), April 2000, pp. 359365.

[32] S. P. Costa, L. van den Engel-Hoek, A. F. Bos, "Sucking and swallowing in infants and diagnostic tools." Journal of perinatology: official journal of the California Perinatal Association, 28(4), 2008, pp. $247-57$.

[33] M. A. Qureshi, F. L. Vice, V. L. Taciak, J. F. Bosma, I. H. Gewolb, "Changes in rhythmic suckle feeding patterns in term infants in the first month of life", Developmental medicine and child neurology, 44(1), 2002, pp. 34-9.

[34] L. Jain, E. Sivieri, S. Abbasi, and Vinod K, Bhutani , "Energetics and mechanics of nutritive sucking in the preterm and term neonate,"Journal of pediatrics, 1987, Vol. 111, pp.894-8.

[35] O. P. Mathew, "Breathing patterns of preterm infants during bottle feeding: role of milk flow", Journal of pediatrics, vol. 119, no. 6, pp. 960-5, Dec. 1991.
[36] M. Taki, K. Mizuno, M. Murase, Y. Nishida, K. Itabashi, Y. Mukai, "Maturational changes in the feeding behaviour of infants - a comparison between breast-feeding and bottle-feeding", 2010, Acta paediatrica , 99(1), 2010, pp. 61-7.

[37] S. Fadavi, I. C. Punwani, L. Jain, D. Vidyasagar, "Mechanics and energetics of nutritive sucking: a functional comparison of commercially available nipples", The Journal of pediatrics, 130(5), 1997, pp. 740-5.

[38] W. C. Lang, N. R. M. Buist, A. Geary, S. Buckley, E. Adams, A. C. Jones, S. Gorsek, "Quantification of intraoral pressures during nutritive sucking: methods with normal infants", Dysphagia, 26(3), 2011, pp. $277-86$.

[39] S. Cecchini, E. Schena, S. Silvestri, "An open-loop controlled active lung simulator for preterm infants", Med. Eng. \& Phys., Vol. 33(1), 2011, pp. 47-55.

[40] C. Lau, R. J. Schanler, "Oral feeding in premature infants: advantage of a self-paced milk flow", Acta paediatrica, 89(4), 200, pp. 453-9.

[41] E. Tamilia, F. Taffoni, E. Schena, D.Formica, L.Ricci, E.Guglielmelli, " A Novel Ecological Method for the estimation of nutritive sucking effiviency in newborns: measurement principle and experimental assessment" accepted for publication in the International Conference of the IEEE Engineering in Medicine and Biology Society (EMBC '13).

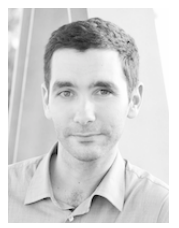

Fabrizio Taffoni received the Ph.D. degree in biomedical engineering from the Università Campus Bio-Medico di Roma (Rome, Italy), in 2009. From 2009 to 2012, he has been a Postdoctoral fellow with the Laboratory of Biomedical Robotics and Biomicrosystems at Università Campus Bio-Medico di Roma where he serves as tenuretrack assistant Professor of Bioengineering from 2012. His research interests, at the intersection between developmental neuroscience, bioengineering and mechatronic, are focused on the design and development of new platforms, tools, and methods for ecological assessment of motor development.

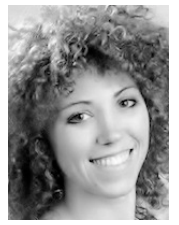

Eleonora Tamilia received the M.S. degree in medical engineering from the University of Rome "Tor Vergata", Rome, Italy, in 2010. She is currently a $\mathrm{PhD}$ student in biomedical engineering at the Università Campus BioMedico di Roma, where she started working in the Laboratory of Biomedical Robotics and Biomicrosystems in January 2012. Her current research interests include: devices and methods for infants' neurodevelopment assessment, specifically focused on newborn's sucking behavior; analysis of experimental data on children's behavior during neurodevelopment.

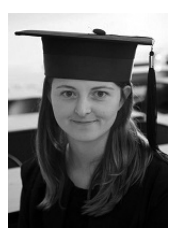

Maria Rosaria Palminteri received the Master degree in biomedical engineering from the Università Campus Biomedico di Roma in 2012. During her Master thesis, she worked in the laboratory of biomedical robotics and Biomicrosystems on the design and development of new methods and tools for non obtrusive assessment of suction in infants. She is currently working as a R\&D Engineer for CRAD Imaging $A B$ in Sweden. Her interests are in the field of medical devices industry and engineering design.

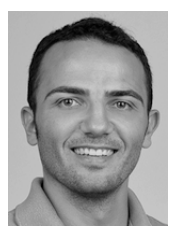

Emiliano Schena received the Ph.D. degree in biomedical engineering from the Università Campus Bio-Medico di Roma (Rome, Italy), in 2009. He is currently Researcher in Mechanical and Thermal Measurements at Università Campus Bio-Medico di Roma. His current research interests include sensors and transducers for mechanical and thermal measurements in the biomedical and clinical field, fiber optic-based measurement systems, and respiratory rehabilitation.

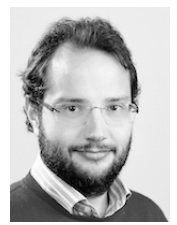

Domenico Formica received the B.S. and M.S. degrees in biomedical engineering and the Ph.D. degree in bioengineering from the Università Campus Bio-Medico, Rome, Italy, in 2002, 2004, and 2008, respectively. From 2008 to 2010, he has been a Postdoctoral Fellow with the Laboratory of Biomedical Robotics and Biomicrosystems, Università Campus Bio-Medico, where he is currently tenure-track assistant Professor of Bioengineering. His 
This is the author's version of an article that has been published in this journal. Changes were made to this version by the publisher prior to publication.

The final version of record is available at http://dx.doi.org/10.1109/JSEN.2013.2271585

\section{IEEE SENSORS}

current research interests include the areas of mechatronic technologies for the study of human motor control, with particular attention to neurodevelopment, quantitative algorithms for clinical assessment of patients with neuromuscular disorders, and novel robotic devices for rehabilitation motor therapy after neurological injury, with special attention to the issue of interaction control.

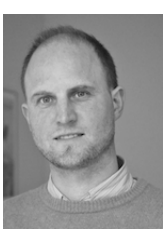

Jonathan Delafield-Butt received his $\mathrm{Ph} . \mathrm{D}$. degree in Developmental Neurobiology from the University of Edinburgh Medical School, Edinburgh, Scotland, in 2003. He is currently Lecturer in Early Years at the University of Strathclyde. His research interest is in early infant psychomotor development with application in improving understanding and treatment of developmental psychopathology, socioemotional care, and learning.

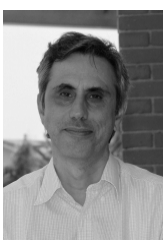

Flavio Keller received the postgraduate degree in neuropharmacology from the University of Zurich in 1982. $\mathrm{He}$ is currently full professor of Physiology at Università Campus Bio-Medico di Roma. He is Head of the Laboratory of Developmental Neuroscience and Neural Plasticity. His main research interests are in the fields of developmental neuroscience and neurobiology (specifically the neurobiology of sensorimotor systems), medicine, and biochemistry. He has author/co-author of more than 80 works appeared on peer-reviewed international journals, conference proceedings and books.

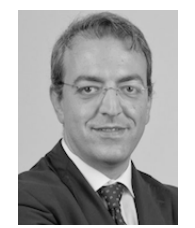

Sergio Silvestri received the Ph.D. degree in mechanical and thermal measurements from Cagliari University, Cagliari, Italy, in 2001. He is currently an Associate Professor of Measurements and Biomedical Instrumentation at the University Campus Bio-Medico of Rome, Rome, Italy. His research interests include sensors for biomedical devices and instrumentation, and minimally invasive and non-invasive medical measurement systems.

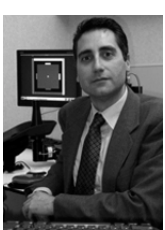

Eugenio Guglielmelli received the degree in Electronics Engineering and the $\mathrm{PhD}$ in Biomedical Robotics from the University of Pisa, Italy, in 1991 and in 1995. He is currently Full Professor of Bioengineering at Campus BioMedico University (Roma, Italy) where he serves as the Head of the Laboratory of Biomedical Robotics and Biomicrosystems, that he founded in 2004. His main current research interests are in the fields of human-centred robotics, biomechatronic design and biomorphic control of robotic systems, and in their application to robot-mediated motor therapy, assistive robotics, and neurorobotics. He is author/co-author of more than 170 papers appeared on peer-reviewed international journals, conference proceedings and books. $\mathrm{He}$ is co-inventor of 3 patents and co-founder of 4 spin-off companies. He served as Associate Editor of the IEEE Robotics \& Automation Magazine, and he serves as Associate Editor of the IEEE Transactions on Robotics. He also serves as Editor-in-Chief of the Springer Series on Biosystems and Biorobotics. 